\title{
Cataract and Coexistent Glaucoma: A Therapeutic Dilemma
}

\author{
Shibal Bhartiya, Harinder S Sethi, Neha Chaturvedi
}

Glaucoma Facility, Dr RP Center for Ophthalmic Sciences, All India Institute of Medical Sciences, New Delhi, India

\begin{abstract}
Glaucoma and cataract are often coexistent in the elderly population. This review provides a comprehensive overview of the preferred practice pattern in this subgroup of patients. Surgical protocols, techniques and diagnostic modalities are discussed comprehensively to enable the glaucoma surgeon to arrive at a management algorithm.
\end{abstract}

\section{INTRODUCTION}

The prevalence of both, glaucoma and cataract increases with age, and thus one often finds that they are coexistent in the elderly patient population. ${ }^{1-3}$ The association of glaucoma with cataracts has become more frequent because of increase in life expectancy and the increased risk of cataract development in the patients with glaucoma.

Moreover, the presence of cataract can affect the ability to assess glaucoma progression, and cataract extraction affects the intraocular pressure and effectiveness of glaucoma surgery. On the other hand, glaucoma surgery significantly increases the risk for the development of cataract. For this reason, and to reduce the trauma and the time taken for patient rehabilitation, the prevailing trend is to perform a combined procedure, taking care of both pathologies simultaneously. Recent developments in bimanual small incision phacoemulsification, ${ }^{4-8}$ the latest improvements in trabeculectomy and nonpenetrating filtering surgery and implant drainage devices ${ }^{9}$ have favored this trend for doing a combined surgery.

The goal of treatment in a glaucoma patient with cataract, is to achieve an adequate long-term control of intraocular pressure (IOP), avoid postoperative IOP spikes which are deleterious to the health of the optic nerve head, obtain an optimal visual rehabilitation and improve the quality of life of the patient. The other important things to keep in mind include minimizing surgical trauma and risk, and ensuring that the procedure is cost effective. With these parameters determining the choice of surgery, it is evident that combined procedures are fast gaining popularity.

Cataract surgery alone has significant effects on the intraocular pressure. Following an early rise in the intraocular pressure, the IOP tends to fall in the long run. Though significant, the magnitude of the change is small, averaging around 2-4 mm Hg and one cannot depend on this as a means of lowering the IOP. The effect of cataract extraction in lowering IOP is much higher in chronic angle closure glaucoma patients than in patients with open angle glaucoma as cataract surgery can result in opening of the iridocorneal angle and reduction of IOP obviating the need for topical medications, without a filtering surgery.

The combined surgical technique of phacotrabeculectomy has become the standard technique for management of eyes with coexistent visually significant cataract and glaucoma. ${ }^{10-12}$

Phacotrabeculectomy can be performed as a single site surgery with both phacoemulsification and trabeculectomy performed from the same site with insertion of a rigid PMMA IOL (Figs $1 \mathrm{~A}$ to $\mathrm{G}$ ) or a foldable IOL (Figs $2 \mathrm{~A}$ to $\mathrm{K}$ ). The second option is a two site surgery, which entails performing a temporal phacoemulsification and a superior trabeculectomy (Figs 3A to I). Separating the two incisions may decrease the inflammation and subsequent fibrosis induced by the surgery leading to a better survival of the filtering bleb.

Microincision cataract surgery (MICS) or Phakonit (implying phacoemulsification performed with a needle) is a recently introduced bimanual technique ${ }^{4-8}$ that permits phacoemulsification via sub 1-1.2 mm incisions (Figs 4A to D). The basic principle is to separate the irrigation from the phacoemulsification handpiece and use an irrigating chopper to maintain the anterior chamber. The advantages of MICS include an astigmatically neutral, safer, closed chamber surgery via smaller incisions and rapid visual restoration with minimal postoperative inflammation. The combined two site surgery by this technique can be performed with surgeon sitting superiorly, i.e without the need to change position during the surgery.

The most important step before operating on a patient with cataract and glaucoma is the preoperative evaluation and decision making regarding the type of surgery to be performed.

\section{PREOPERATIVE EVALUATION}

In addition to the routine evaluation conducted for any cataract patient, patients with a coexistent glaucoma require evaluation of the ongoing medical therapy, diurnal IOP control on medication, corneal endothelial specular count, gonioscopy, 
stereoscopic disk evaluation preferably with +78 D or 90 D lens and visual fields (if possible). Conjunctival inflammation due to topical drug therapy, a low corneal endothelial count, miotic pupil, poor response to mydriatics, posterior synechiae, weakened zonules (esp. in eyes with pseudoexfoliation) and uncontrolled IOP are some of the important factors which may complicate the surgical procedure, compromising the postoperative outcome.

Drugs such as pilocarpine and prostaglandin analogs should be stopped atleast 2 weeks prior to the surgery. Iris hooks may be required for intraoperative pupillary dilatation, especially in eyes with primary angle closure glaucoma and endocapsular rings should be kept ready if surgery is being planned in a case with pseudoexfoliation syndrome.

The decision to do a cataract surgery alone, or a combined procedure or to do a filtering surgery alone is decided by evaluation of the following factors:

- IOP control on current treatment

- Target IOP for the patient

- Number of medications needed to achieve target IOP

- Extent of glaucomatous damage (disk and visual fields)

- Type of glaucoma (open angle or angle closure)

- Compliance to medical therapy

- Allergic reactions/significant side effects of topical therapy

- Socioeconomic status of the patient

- Access to medical care facilities

- Effect of disease on quality of life of the patient.

\section{OPTIONS FOR SURGICAL MANAGEMENT}

Even though the ideal protocol for surgery is still controversial, combined surgery to tackle both the cataract and glaucoma is a widely accepted modality of treatment. ${ }^{13-16}$ The advent of phacoemulsification has further allowed the reduction of incision size resulting in a decrease in the complication rate. The advantages of such a combined approach include rapid visual rehabilitation, one time surgery, a reduced cost and increased patient comfort.

Since combined surgical procedures have reduced long time success rates as compared to that of trabeculectomy alone, with increased incidence of postoperative inflammation, careful case selection is important. Combined surgery is best avoided in patients with secondary glaucoma, extensive conjunctival scarring, normal pressure glaucoma, advanced glaucomatous optic neuropathy and previous failed trabeculectomy. The other surgical option in such patients would be to perform a staged procedure with a trabeculectomy followed by cataract surgery in the second sitting. We recommend the use antimetabolites (Mitomycin C 0.2-0.4 mg/ml) routinely in combined surgery.

\section{THERAPEUTIC OPTIONS}

The following treatment options are available when dealing with a case with coexistent cataract and glaucoma:
1. Cataract surgery alone.

2. Laser trabeculoplasty followed by cataract extraction.

3. Filtering procedure followed by cataract extraction at a later date.

4. Simultaneous cataract and glaucoma surgery-Combined extraction

a. Extracapsular cataract extraction + Trabeculectomy

b. Manual small incision cataract surgery (SICS) + Trabeculectomy

c. Standard phacoemulsification + Trabeculectomy

i. Single site

ii. Two site

d. MICS (Phakonit) + Trabeculectomy ${ }^{17}$

e. Endoscopic cyclophotocoagulation + Cataract surgery ${ }^{18}$

f. Deep Sclerectomy + Cataract surgery

g. Glaucoma drainage device + Cataract surgery.

\section{Cataract Extraction Alone}

It is probably the treatment of choice in patients on a low-dose, well-tolerated medical regimen and with early or no glaucomatous optic nerve damage. It is not advisable in patients with moderate/ advanced glaucomatous field damage because even slight postoperative rise of intraocular pressure (IOP) can have a deleterious effect on the remaining field of vision, the "washout syndrome". Its incidence may be minimized by using perioperative beta blockers and oral carbonic anhydrase inhibitors to blunt any postoperative IOP spikes. On the other hand, cataract extraction in a patient of chronic angle closure glaucoma may result in lowering of IOP, allowing the withdrawl of antiglaucoma drugs.

These patients should be followed up on a regular basis, as their glaucoma control can deteriorate any time in the future, requiring initiation/enhancement of medical therapy or even filtering surgery.

Therefore, if the IOP is well controlled on a single topical drug with early glaucomatous optic nerve damage, cataract surgery alone can be performed.

\section{Surgical Caveats}

- A dispersive viscoelastic device such as chondroitin sulfate or viscoadaptive like Healon 5 (23 mg/ml of sodium hyaluronate) should be used protect the compromised corneal endothelium of glaucoma patients. In addition chilled BSS plus with glutathione may be used to maintain integrity of corneal endothelial cells during surgery.

- Poor pupil dilatation may be encountered especially in angle closure glaucomas and patients on long-term pilocarpine therapy. This may require additional surgical manipulations to achieve optimal visualization. Stretching maneuvers (bimanual stretch pupilloplasty with two Sinsky or V/Y 
hooks) or the use of iris hooks or rings for pupillary dilatation are therefore recommended.

- The weakness of the zonules and the increased contraction of the capsule in pseudoexfoliative glaucoma requires additional intraoperative care and the need to use a capsular tension ring.

- In glaucomatous eyes and especially in pseudoexfoliation syndrome, there is a tendency for enhanced breakdown of the blood-aqueous barrier, further aggravated by the use of pilocarpine and prostaglandins. Intense topical steroid therapy for a longer period of time may be required in the postoperative period, with occasional use of systemic steroids.

- The surgeon has to be extracautious in these cases as a posterior capsule rent with vitreous loss will aggravate the glaucomatous process, leading to inflammation and a rise in IOP.

- During the course of cataract surgery, care should be taken to completely remove any viscoelastic used. This is especially important if Healon 5 has been used as it can cause severe postoperative IOP elevation. This needs to be aspirated using high flow rate settings in the irrigation and aspiration mode of the phaco machine if working on the peristaltic system and using high vacuum settings if using venturi systems. Also it needs to be aspirated from behind the IOL to ensure complete removal as it may not be aspirated using the routine rock and roll maneuver.

- One has to be cautious against significant intraocular pressure spikes in the immediate postoperative period which may further compromise a glaucomatous optic nerve. These patients should be put on ocular hypotensive therapy in the postoperative period to prevent any IOP spike.

In extracapsular cataract extraction (ECCE) or manual SICS through a superior limbal approach, the limbal conjunctiva is incised during the surgery. The conjunctiva in this region is thus prone to scarring, which may preclude creation of a filtering bleb in this location.

The advent of phacoemulsification, especially the clear corneal approach for access to the cataractous lens has revolutionalised cataract surgery. Phacoemulsification is performed with a significantly smaller incision than ECCE and therefore induces smaller amounts of astigmatism and does not affect the conjunctiva. Clear corneal, especially temporal, phacoemulsification cataract surgery is the preferred approach to maximize the options available for future glaucoma surgery. Microincision cataract surgery (MICS) is an equally good option for cataract extraction in such cases.

\section{Laser Trabeculoplasty Followed by Cataract Extraction}

Laser trabeculoplasty followed by cataract extraction is another therapeutic alternative. Although Argon laser (ALT) has been commonly used, SLT, diode laser, double frequency Nd:YAG laser, krypton laser, etc may also be used. This decreases the risk of immediate elevation of IOP in the postoperative period and may also reduce the requirement of antiglaucoma medications both prior to and following cataract surgery. Complications following ALT include hemorrhage from the trabecular meshwork during treatment, formation of peripheral anterior synechiae, uveitis and elevation of IOP. There is a decrease in the success rate of ALT with time and so these patients need to be on a regular follow-up.

Another option is to do a Selective Laser Trabeculoplasty (SLT) followed by cataract surgery. This is a new technique for laser trabeculoplasty which uses a $\mathrm{Q}$ switched double frequency (532 nm) YAG laser with a nanosecond pulse which does not cause any thermal damage and is selectively absorbed by the pigment granules in the trabecular meshwork. The advantage of SLT is that there is no thermal effect and therefore it does not lead to scarring and can be repeated unlike ALT.

\section{Filtering Surgery Followed by Cataract Extraction at a Later Date}

When glaucoma is uncontrolled in spite of maximal tolerable medical therapy and/or laser trabeculoplasty, a trabeculectomy should be performed alone. This is also the case in eyes with advanced glaucoma that require a very low target pressure and eyes with secondary glaucoma associated with uveitis or neovascularization. Cataract extraction can be performed through a temporal clear corneal at a later date (preferably after 3 months). ${ }^{19,20}$ One can use a subconjunctival injection of 5 FU (5 mg/0.1 ml) away form the site of surgery and the bleb to prevent any subsequent compromise of the filtering bleb after completion of cataract surgery. Care needs to be taken that 5 FU does not enter into the anterior chamber via leakage during subconjunctival injection. It should preferably be injected with a $30 \mathrm{G}$ needle and any leaking 5 FU should be immediately soaked in via a cotton swab/Merocel sponge placed at the site of the injection by the assistant. There is a concern in such cases that cataract surgery may trigger inflammation and stimulate a fibroblastic response in the bleb leading to bleb failure.

\section{Simultaneous Cataract and Glaucoma Surgery- Combined Extraction}

Indications for a combined procedure include:

- When in spite of maximal tolerable topical medical therapy and/or laser trabeculoplasty, glaucoma control is poor in a patient with mild/moderate glaucoma.

- When the patient doesn't tolerate the medical therapy or is not compliant with antiglaucoma medications.

- When the patient cannot afford long-term medical therapy.

- Patients of advanced glaucomatous damage who cannot tolerate postoperative IOP spike. 
- Uncontrolled glaucoma, but an urgent need to restore vision or when two separate surgeries are not feasible.

A combined procedure should be performed in eye with advanced glaucomatous damage with significant cataracts, even if IOP is well-controlled because even a transient rise of IOP postoperatively can threaten the residual field of vision.

When combining glaucoma surgery with cataract extraction, the surgery becomes technically more difficult than either surgery alone, there is more postoperative inflammation, the bleb formation is less reliable and the lowering of IOP may not be adequate to the amount of glaucomatous damage (i.e one may not be able to achieve the target pressure for a particular patient).

Two site surgery, separating the phacoemulsification and the trabeculectomy site has the theoretical advantage of reducing inflammation at the site of the filter and thereby decrease in the stimulus for the subsequent fibroblastic response. Standard two site phacotrabeculectomy requires two separate incisions, one for the cataract surgery and the other for the ostium under the scleral flap. In addition, the surgeon needs to adjust his position intraoperatively along with that of his assistants and equipments and the operating microscope (i.e superior for trabeculectomy and temporal for phacoemulsification). Phacoemulsification may now be performed with the use of AQUALASE (warm water jets) which leads to a much quieter eye postoperatively.

MICS/Phakonit offers the dual advantage of performing a coaxial bimanual closed chamber surgery and removing the cataract via an incision which is one-third the size of a routine phacoemulsification incision (1-1.2 mm versus a standard 3.2 $\mathrm{mm}$ incision). These ultra small incisions induce less inflammation which is a significant advantage when this surgery is combined with a trabeculectomy. A reduction in postoperative inflammation is likely to reduce the risk of fibrosis and filter failure. Although new ultrathin IOLs (such as the ThinOptx rollable IOL) have been implanted after MICS, ${ }^{21}$ further investigation is required before they are adopted as a standard procedure in cataract surgery.

We have introduced a new technique of combining MICS with trabeculectomy and insertion of a foldable IOL through the trabeculectomy fistula. ${ }^{22}$ This technique offers the additional advantage of implantation of a standard hydrophobic acrylic IOLs with reduced risk of posterior capsule opacification, through the ostium of trabeculectomy incision. The surgeon does not have to enlarge the side port incisions for introducing the IOL.

\section{Surgical Technique}

Although cataract extraction has also been successfully combined with nonpenetrating glaucoma surgery, endoscopic laser ablation and with implant surgeries our experience with these techniques is limited and we routinely do a two site phacotrabeculectomy.

The surgery should be performed under peribulbar anesthesia. Optimal pupillary dilatation is desirable to facilitate cataract extraction. Either a fornix or limbal based conjunctival flap may be used. Care should be taken to preserve conjunctiva so that future filtering surgeries may be possible, if required.

\section{Single Site Phacotrabeculectomy}

This can be done by 2 methods either via creating a scleral flap or via a scleral tunnel.

For a limbal based flap, the conjunctiva is incised 8-9 mm behind the limbus. Wescott's scissors are used to separate the Tenons capsule and extend the conjunctival incision. For a fornix based flap, an incision is made in the conjunctiva at the limbus and extended for a distance of around 7-8 mm. After dissecting the conjunctival flap superiorly, a triangular/ rectangular scleral flap is marked with a sharp blade approximately $5 \mathrm{~mm}$ wide and $5 \mathrm{~mm}$ in height. Dissection is carried out with a steel crescent knife/diamond knife (Fig. 1A) and extended into clear cornea. The use of antimetabolites is recommended. $0.2-0.4 \mathrm{mg} / \mathrm{ml}$ concentration of Mitomycin C is used for 2-3 minutes subconjunctivally using 4-5 sponges .This is followed by a thorough wash with balanced salt solution. The conjunctiva is then reposited back and cataract surgery started.

In case the surgeon prefers the one site technique (Fig. 1A), then entry into the anterior chamber is made under the scleral flap with a $3.2 \mathrm{~mm}$ keratome (Fig. 1B) and the phacoemulsification (Fig. 1C) completed and the IOL implanted (Fig. 1D), before cutting the block of tissue under the scleral flap (Fig. 1E). A peripheral iridectomy is performed. The iris is reposited by gently stroking the cornea and the scleral flap secured with three 10-0 monofilament sutures (Fig. 1F), releasable/adjustable sutures may also be used. The conjunctival flap is sutured with running 8-0 vicryl sutures if a limbus based flap has been used. A fornix based flap is pulled down and sutured to the limbus with two 8-0 vicryl anchoring sutures and additional 10-0 nylon sutures to ensure that there is no leakage under the flap.

There is another way of performing the single site phacotrabeculectomy through the scleral tunnel (Figs 2A to $\mathrm{K})$. In this technique, scleral tunnel is constructed (Figs 2A to $\mathrm{C}$ ). The phacoemulsification is then performed through it (Figs 2D and E). After implanting IOL (Figs 2F and G), the trabeculectomy window is cut near the inner posterior lip of the tunnel with the help of Kelly's Descemet punch (Figs 2H and I). The tunnel is then sutured or left unsutured depending upon the case and surgeon preference. The scleral tunnel is then covered with the conjunctiva (Figs $2 \mathrm{~J}$ and $\mathrm{K}$ ). 


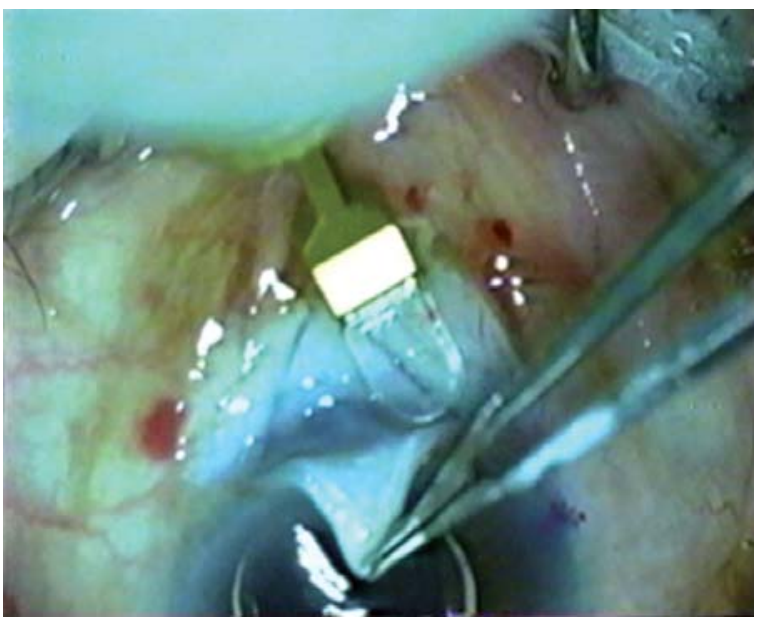

Fig. 1A: Triangular scleral flap underneath a fornix based conjunctival flap

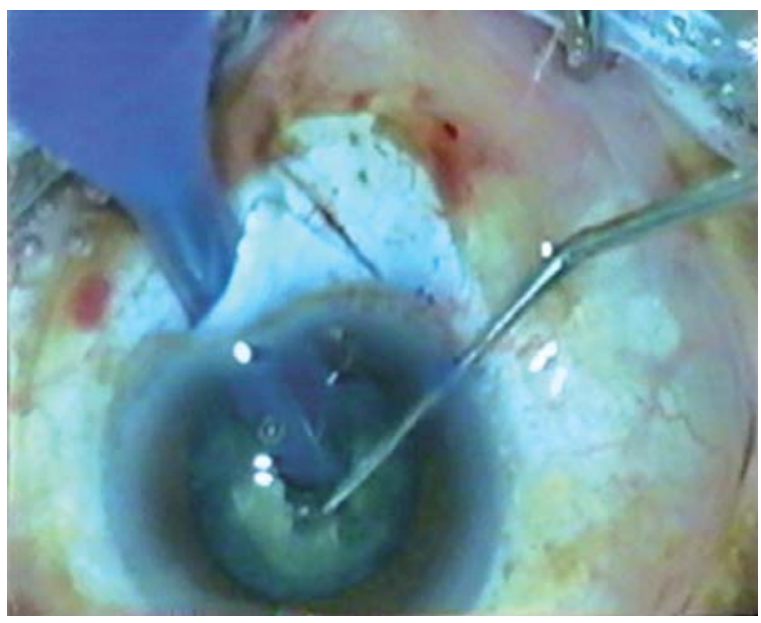

Fig. 1C: Phacoemulsification being performed

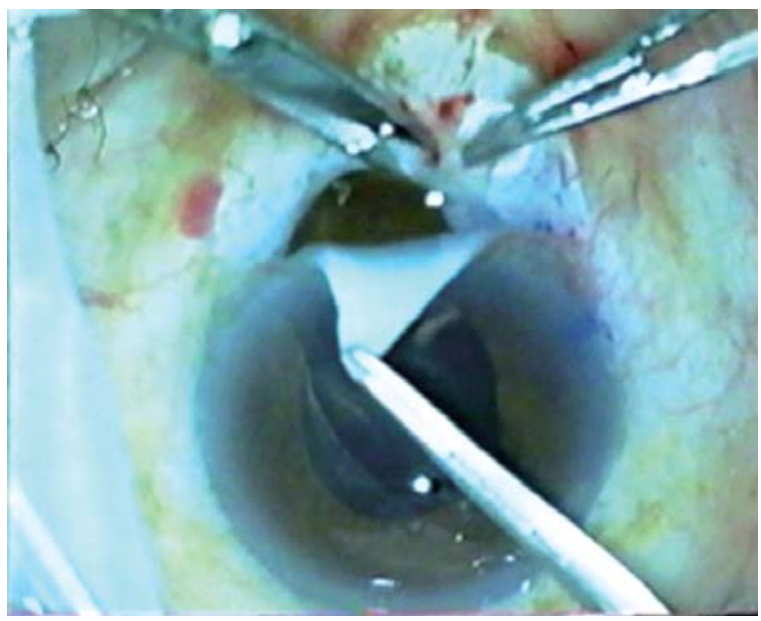

Fig. 1E: Trabeculectomy block being excised under the scleral flap with vanna's scissors

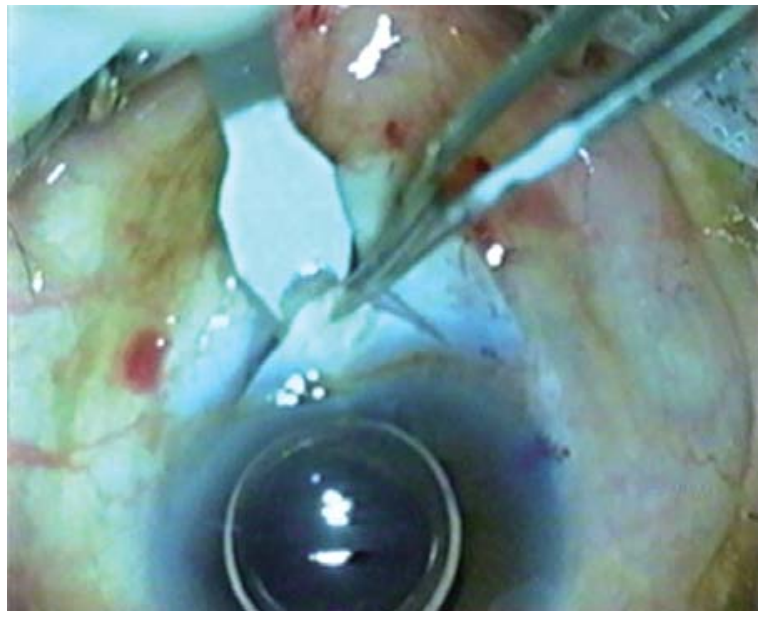

Fig. 1B: Entry into clear cornea under scleral flap for phacoemulsification

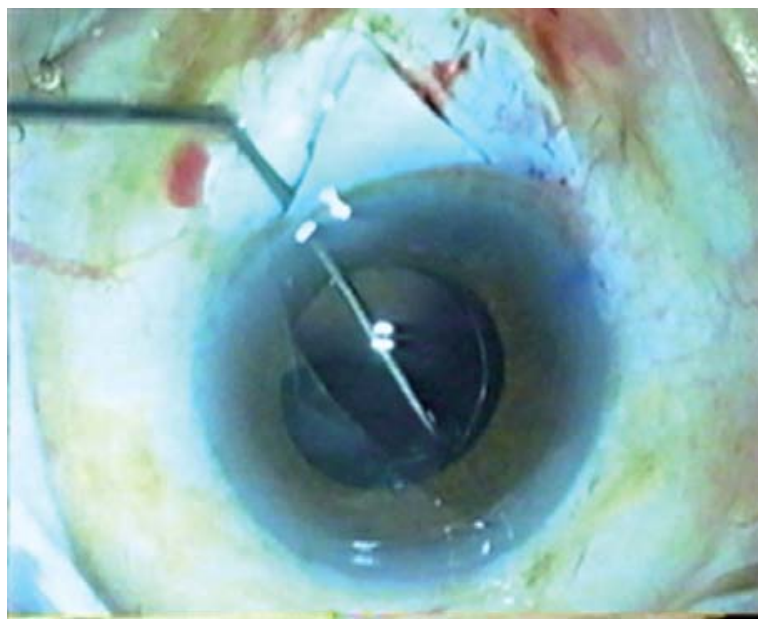

Fig. 1D: PMMA IOL inserted into the capsular bag

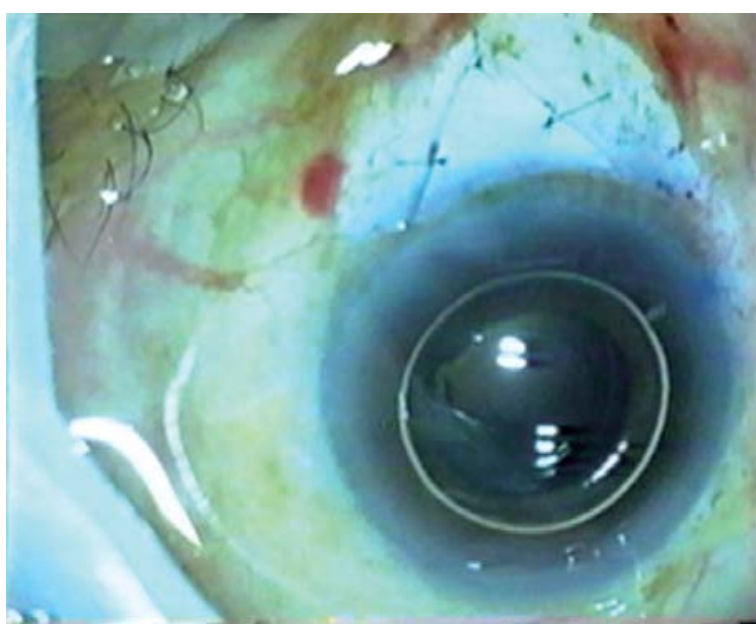

Fig. 1F: Final appearance after closure of the scleral flap

Figs $1 \mathrm{~A}$ to $\mathrm{F}$ : Single site phacotrabeculectomy through a scleral flap 


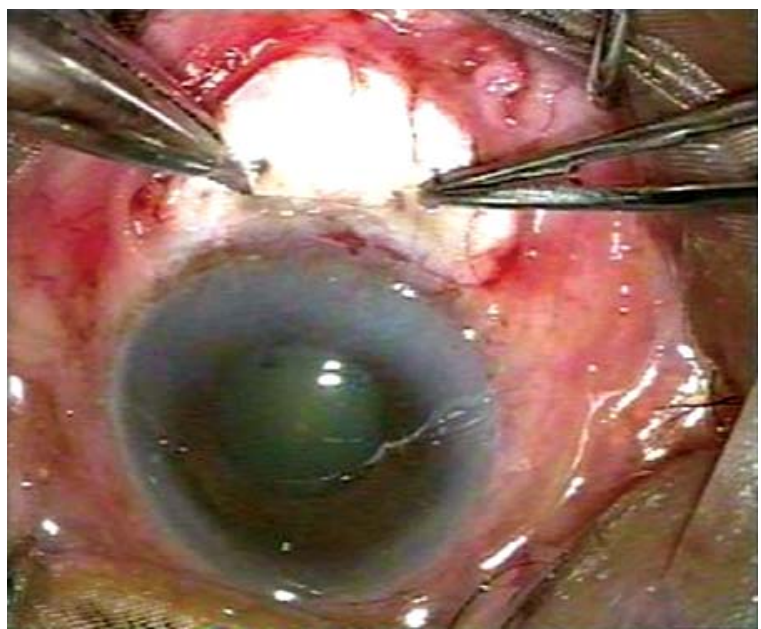

Fig. 2A: Scleral groove being marked with a blade underneath a fornix based conjunctival flap

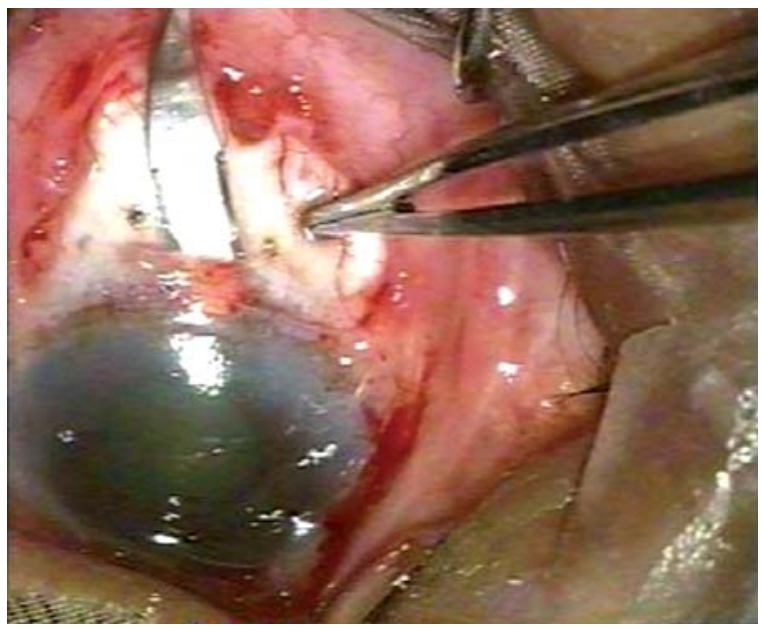

Fig. 2C: Clear corneal entry with a $3.2 \mathrm{~mm}$ keratome

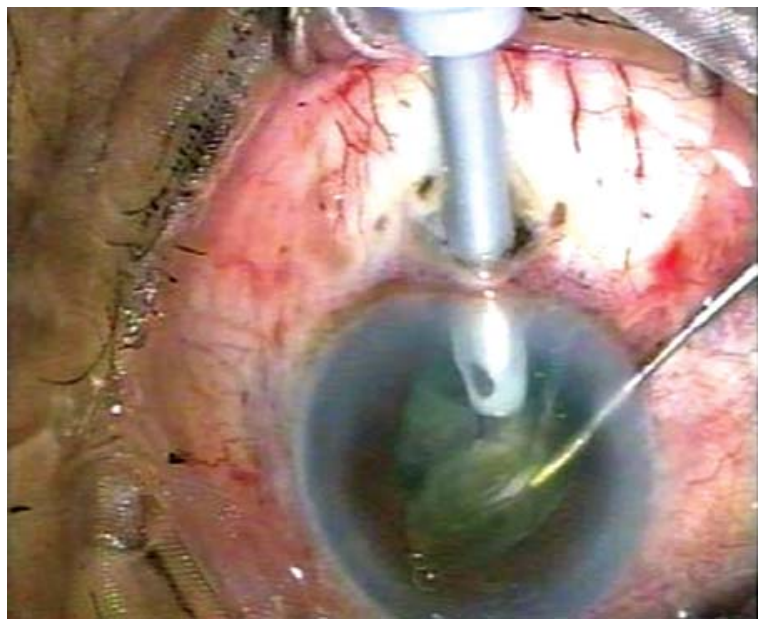

Fig. 2E: Phacoemulsification being carried out

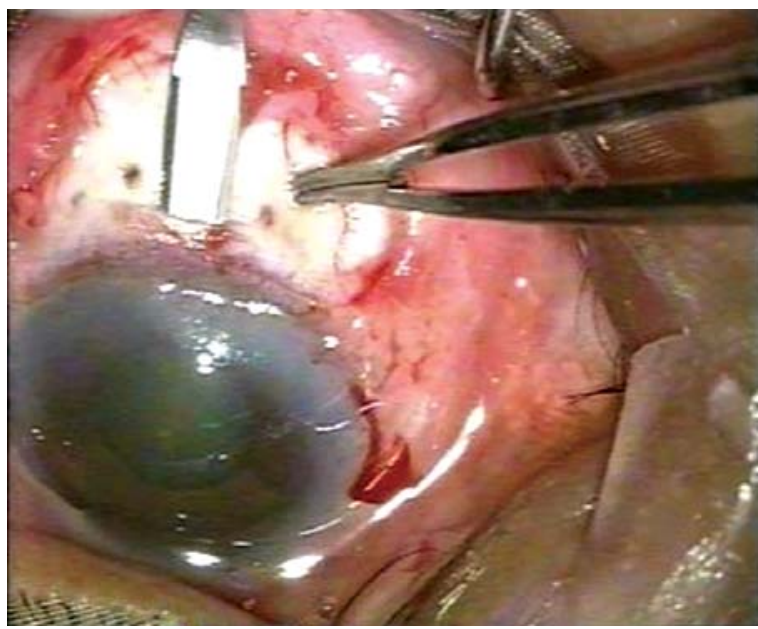

Fig. 2B: Scleral tunnel being fashioned

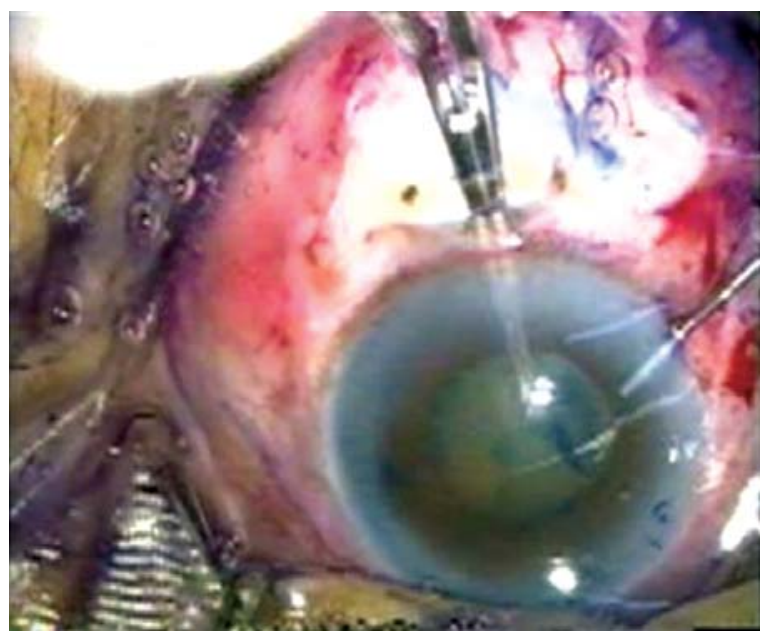

Fig. 2D: Capsulorhexis being performed from the scleral tunnel

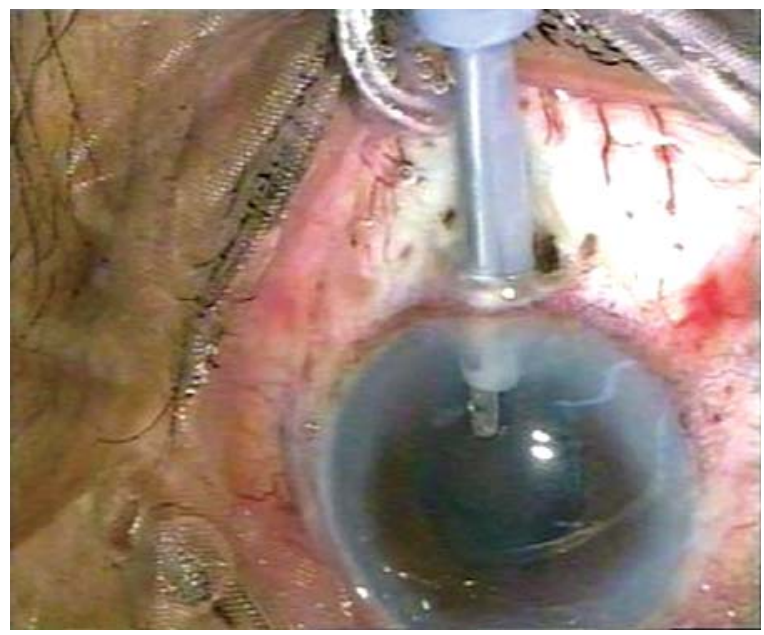

Fig. 2F: Irrigation aspiration being carried out 


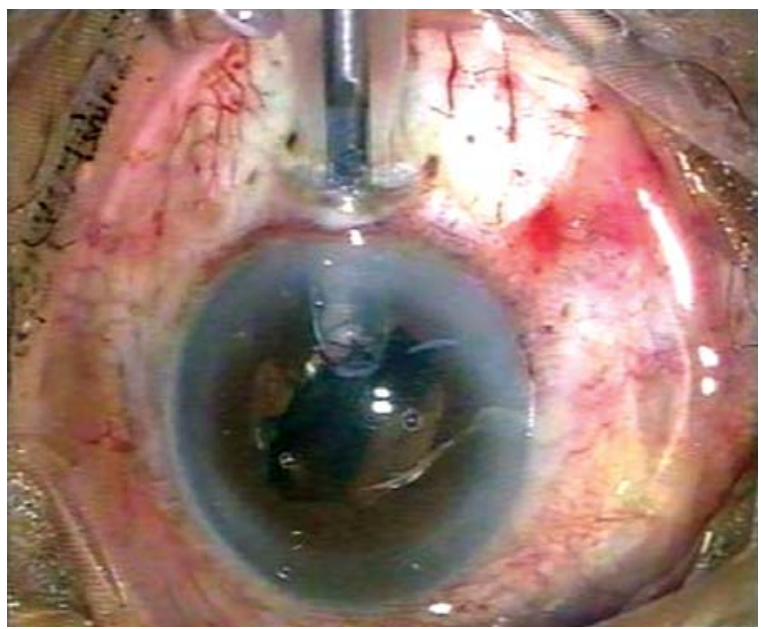

Fig. 2G: Foldable IOL insertion

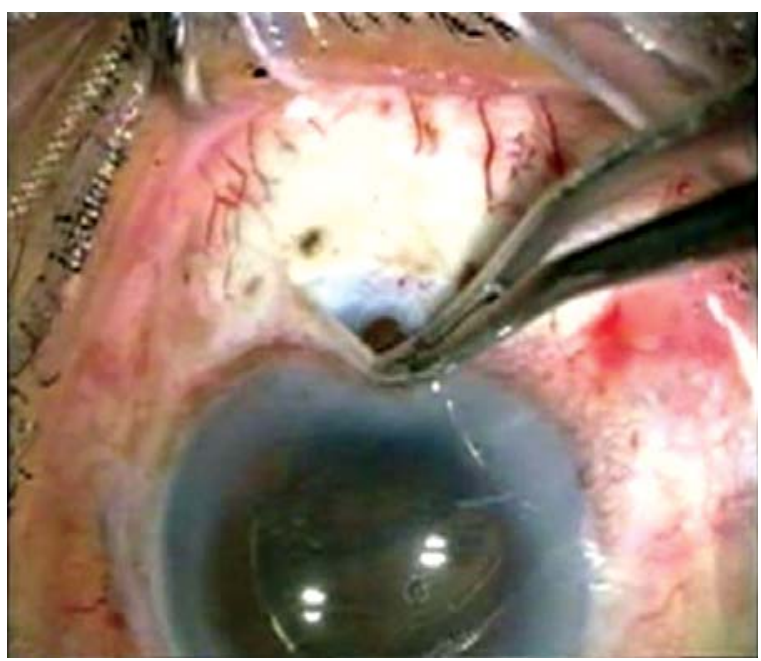

Fig. 2I: Appearance after block excision

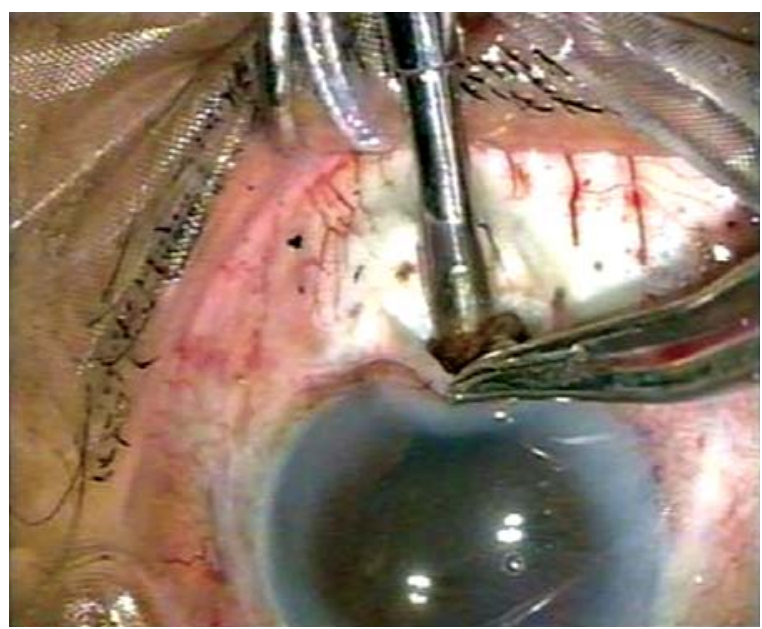

Fig. 2H: Trabecular meshwork being excised with Kelly's descemet punch

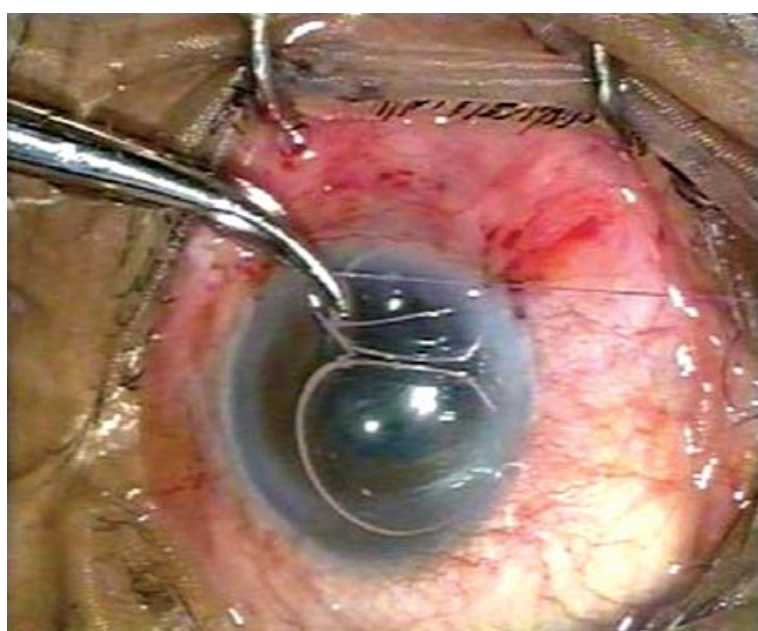

Fig. 2J: Fornix based conjunctival flap being resutured

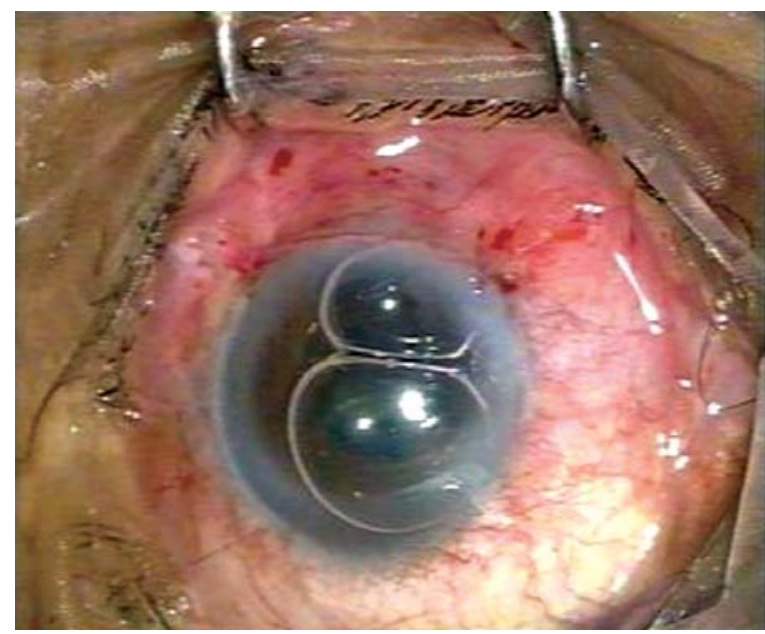

Fig. 2K: Final appearance at completion of surgery

Figs 2A to K: Single site phacotrabeculectomy underneath a scleral tunnel 


\section{Two site Phacotrabeculectomy}

The two site procedure is our preferred mode of surgery as by separating the incisions for phacoemulsification and trabeculectomy, the postoperative IOP control is better than the single site procedure.

If a two site surgery is performed, the surgeon first makes a temporal entry with a 2.75/3.2 mm Keratome or a diamond knife (Fig. 3A) after making a side port entry with a MVR blade. After completing standard phacoemulsification (Fig. 3B), the cortex is removed by irrigation aspiration (Fig. 3C) and foldable IOL implanted under a viscoelastic (Fig. 3D). The viscoelastic should not be removed after IOL implantation. A square edged hydrophobic acrylic IOL is our choice for a phacotrabeculectomy.

The surgeon shifts back to the superior limbus to complete the trabeculectomy. A conjunctival flap is fashioned with Wescott's scissors (Fig. 3E). The bleeders are cauterized with bipolar cautery and a triangular flap $5 \times 5 \mathrm{~mm}$ marked with a super sharp blade (Fig. 3F). The scleral flap is dissected to the level of clear cornea with a crescent knife or a diamond blade. Mitomycin C is used as described previously.

The anterior chamber is entered with a sharp blade or MVR knife, and a block of tissue $3.5 \times 1 \mathrm{~mm}$ is cut with Vanna's scissors. The scleral flap is lifted, the iris pulled out and a peripheral iridectomy performed (Fig. 3G). The scleral flap is closed with two/three 10-0 monofilament sutures (Fig. 3H). The viscoelastic can now be removed via the temporal corneal incision. One 10-0 nylon suture should be applied to the corneal incision to prevent any possibility of leakage if massage of the bleb is required in the postoperative period. A Kelly's descemet punch can also be used for cutting the block and releasable sutures may be used to allow titration of filtration after surgery.

If a limbus based flap has been used then the conjunctiva is closed by a running 8-0 Vicryl suture (Fig. 3I). For a fornix based flap, the conjunctiva is pulled down and secured at the edges with 8-0 Vicryl sutures to the cornea. Additional 10-0 nylon sutures may be placed to achieve water-tight closure. Care should be taken to avoid covering releasable sutures with conjunctiva. In all cases a subconjunctival injection of 0.25 cc dexamethasone $4 \mathrm{mg} / \mathrm{ml}$ and 0.25 cc gentamicin $40 \mathrm{mg} / \mathrm{ml}$ is given at the end of the surgery in the lower fornix and $5 \%$ povidone-iodine sprayed over the surgical field.

Alternatively some surgeons prefer making conjunctival and scleral flap without anterior chamber entry before phacoemulsification. The scleral flap dissection is easier in a closed globe with normal pressure and without the chance of anterior chamber leak. The sugeon then shifts to the temporal site to perform phacoemulsification and IOL implantation. After that the surgeon again shifts back to superior location to complete the trabeculectomy.

\section{B. Microincision Cataract Surgery Combined with Trabeculectomy}

This is a new technique advocated by the author in which cataract surgery is performed through two side ports and trabeculectomy done from the superior site (Figs $4 \mathrm{~A}$ and D). It allows for a closed chamber surgery with decreased endothelial cell loss, ${ }^{23-26}$ and less use of phaco energy and fluids. ${ }^{27}$

\section{Trabeculectomy Alone Followed by Second Stage Cataract Surgery}

The success rate for combined procedures is lower than that reported for trabeculectomy alone. We generally space the two surgeries by 6 months. The trabeculectomy is done superiorly and the cataract removed by a temporal approach to avoid the bleb site. Since there is leakage of fluid from the bleb site, the bottle height should be raised to an adequate amount. The use of a Superpinky should be avoided as it can result in a gross hypotony with shallowing of the anterior chamber. The corneal endothelium should be coated with a dispersive viscoelastic (Viscoat) or a viscoadaptive (Healon 5) to provide maximal protection. One can also assess the functional state of the filtering bleb at the time of cataract surgery when one has injected trypan blue dye into the anterior chamber prior to capsulorhexis. If the bleb is functioning well, then there should be diffuse staining of the filtering bleb. Indeed preliminary data from our study (Trypan blue staining to assess filtration function in trabeculectomized eyes undergoing phacoemulsification. Presented at ASIA ARVO, Singapore, March 2007) show that patients having diffuse, uniform trypan blue staining have the best postoperative IOP control following cataract surgery. Subconjunctival injection of 5-FU or mitomycin drops must be given as there is a risk for bleb failure after the second stage cataract surgery. This can occur because during surgery there is a breakdown of the blood aqueous barrier and inflammation which can subsequently lead to bleb fibrosis.

\section{Postoperative Management}

A close watch for complications is needed in the immediate postoperative period. Due to the iris manipulation, there is an increased chance of severe postoperative uveitis which requires intense topical and sometimes even systemic steroids. Followup examinations are recommended on the first postoperative day, fourth postoperative day and weekly thereafter in the first month. Topical corticosteroids in maximal strength are used two hourly in the first week and tapered gradually to be discontinued by 12 weeks. It is important to remember that since there is more postoperative inflammation following combined phacotrabeculectomy than phacoemulsification, these patients should be kept on topical steroids for a more prolonged duration upto 12 weeks than in patients undergoing phacoemulsification in which the anti-inflammatory medications are usually tapered off within 4 weeks. 


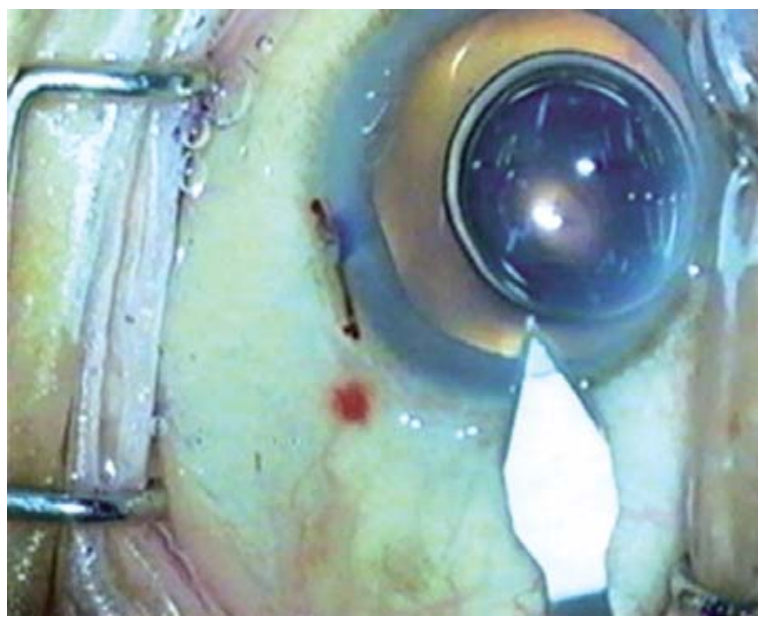

Fig. 3A: Clear corneal tunnel with a $3.2 \mathrm{~mm}$ keratome

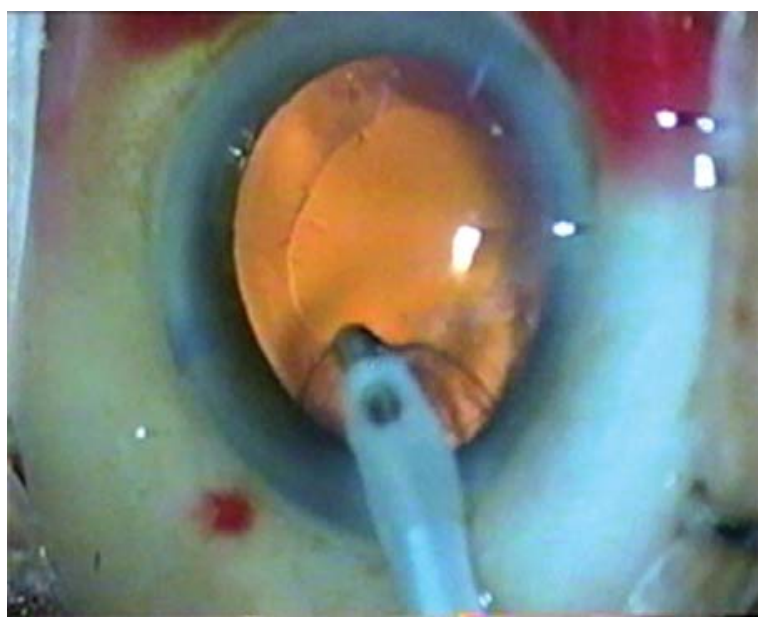

Fig. 3C: Irrigation aspiration and removal of subincisional cortical matter

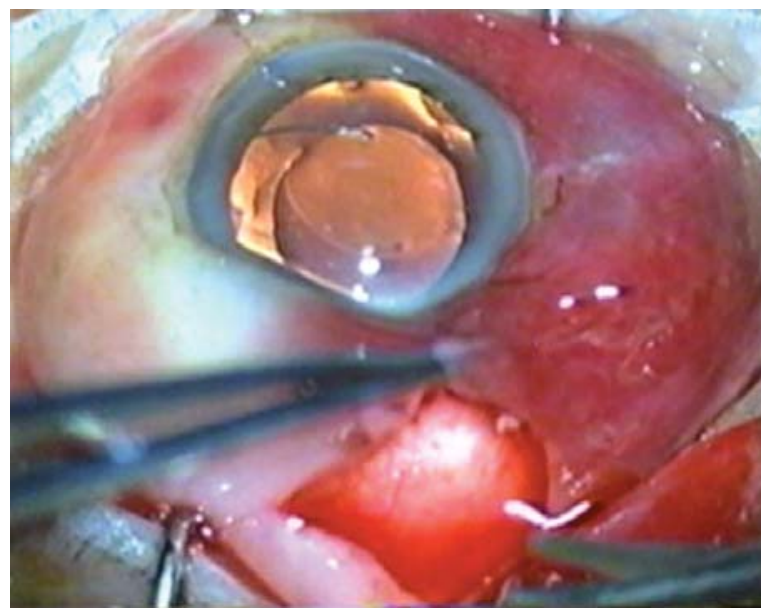

Fig. 3E: Limbus based superior conjunctival flap creation

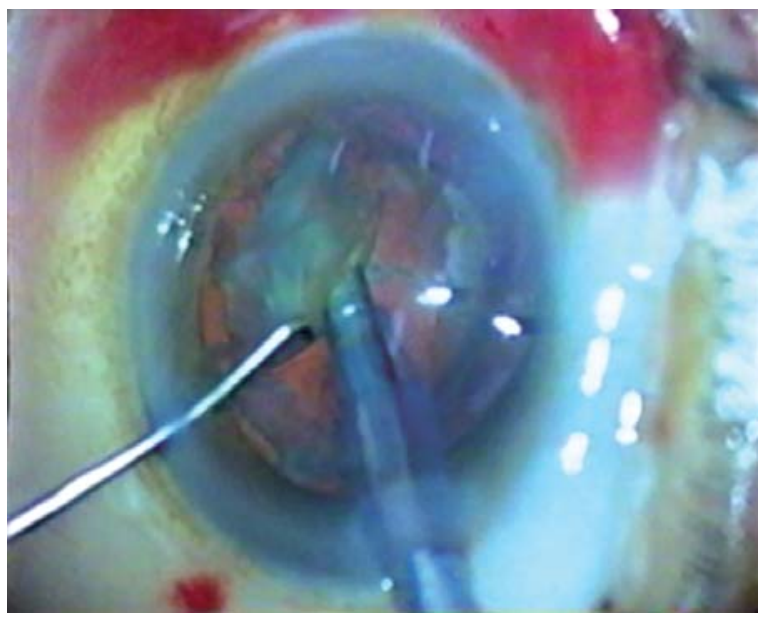

Fig. 3B: Phacoemulsification being carried out

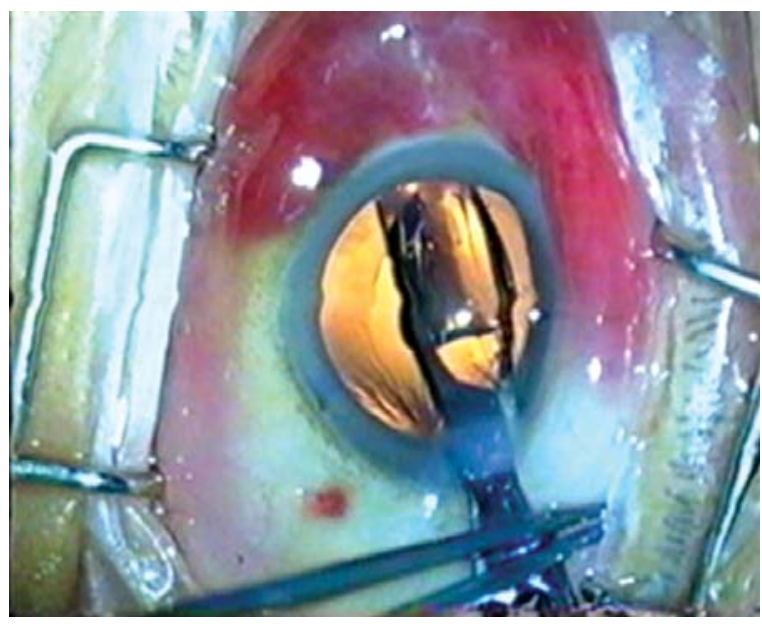

Fig. 3D: Foldable IOL insertion through a holder-folder system

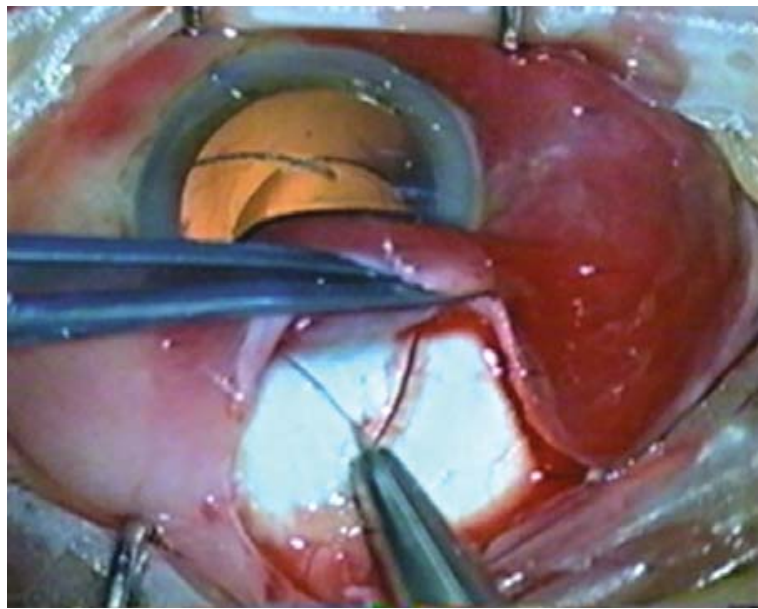

Fig. 3F: Triangular scleral flap being fashioned 


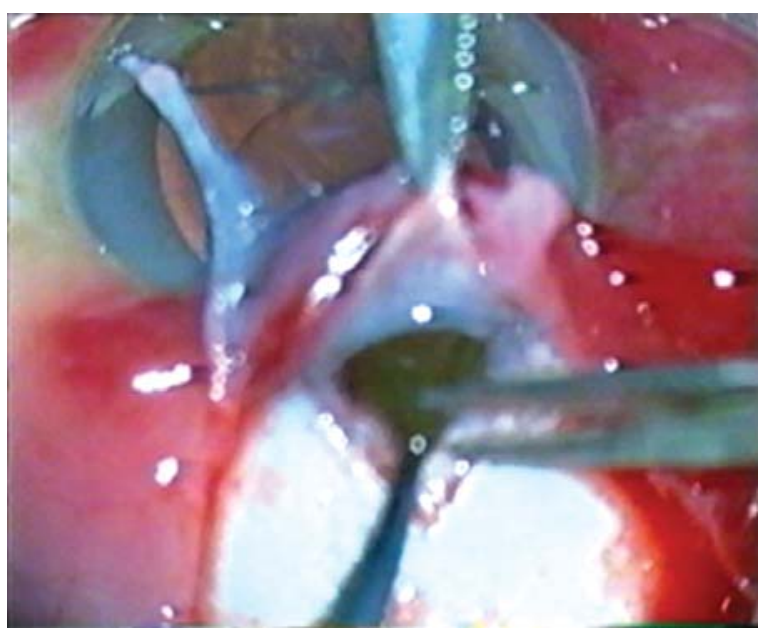

Fig. 3G: Peripheral iridectomy being performed underneath scleral flap to complete the procedure

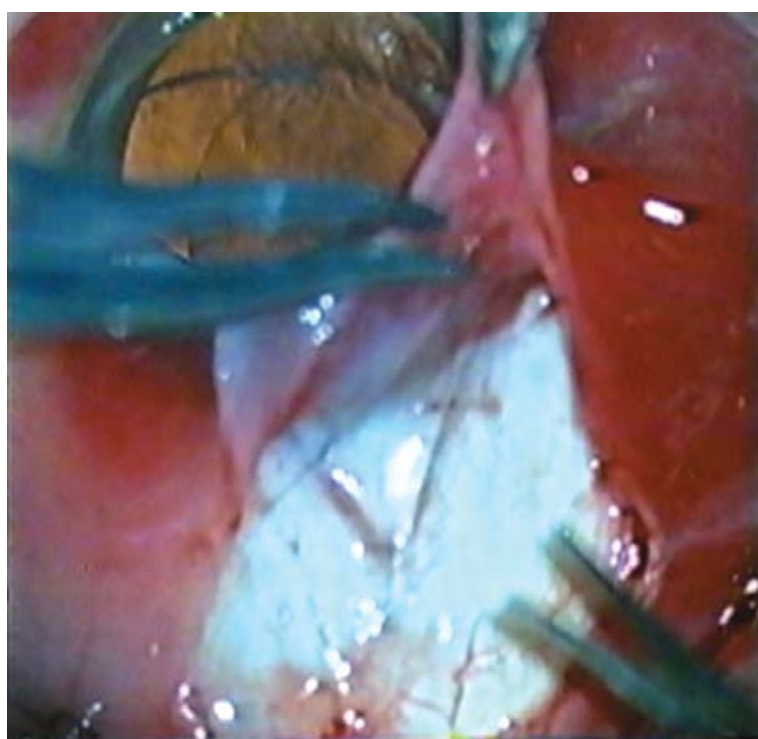

Fig. 3H: Closure of scleral flap with 10-0 nylon sutures

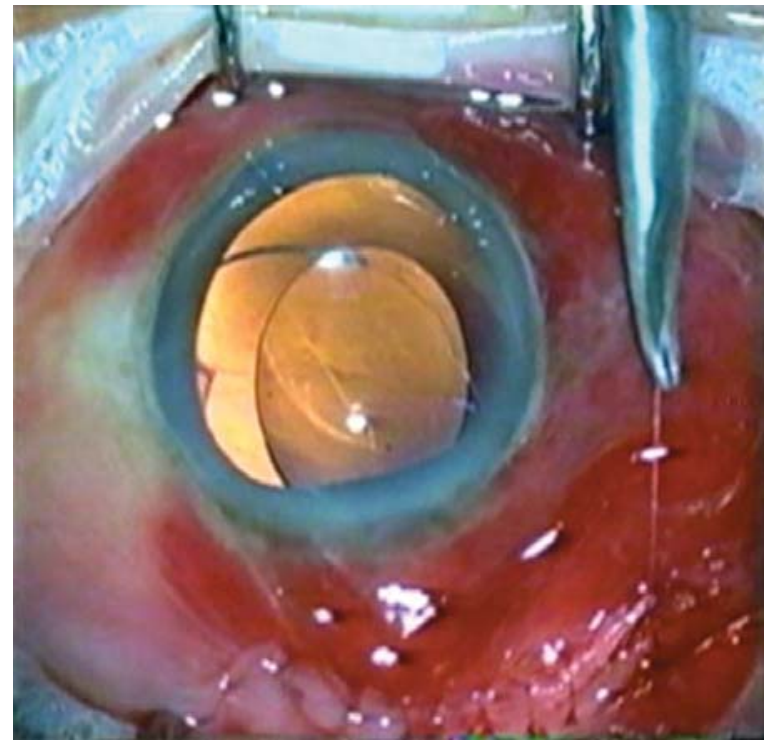

Fig. 3I: Conjunctival flap being closed with running 8-0 vicryl suture

Figs $\mathbf{3 A}$ to I: Two site phacotrabeculectomy

Topical antibiotic drops and short acting mydriatics are also used in the first postoperative month. Should releasable sutures be removed, antibiotic drops are continued till 2 weeks after the suture removal. Dilute pilocarpine therapy (0.25\%) may be tried in patients who complain of glare due to a permanent mydriasis following the use of iris hooks during surgery. These patients require a long-term follow-up as there is always a risk of failure of the filtering surgery with a subsequent rise in the IOP.

\section{Management of Postoperative Complications}

The complication rate of a combined procedure has been reported to be similar to that following trabeculectomy. Vision threatening complications include severe postoperative uveitis, suprachoroidal hemorrhage, hypotony, a flat anterior chamber, raised IOP and the need for a repeat surgery. ${ }^{28}$

1. Postoperative inflammation: Uveitis is generally more severe when the two procedures are combined together. It 


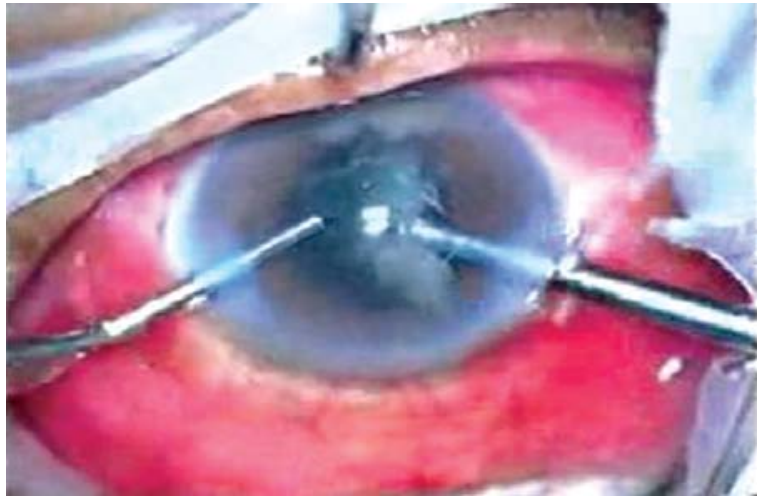

Fig. 4A: Bimanual phacoemulsification being performed- note bare phaco needle and irrigating chopper

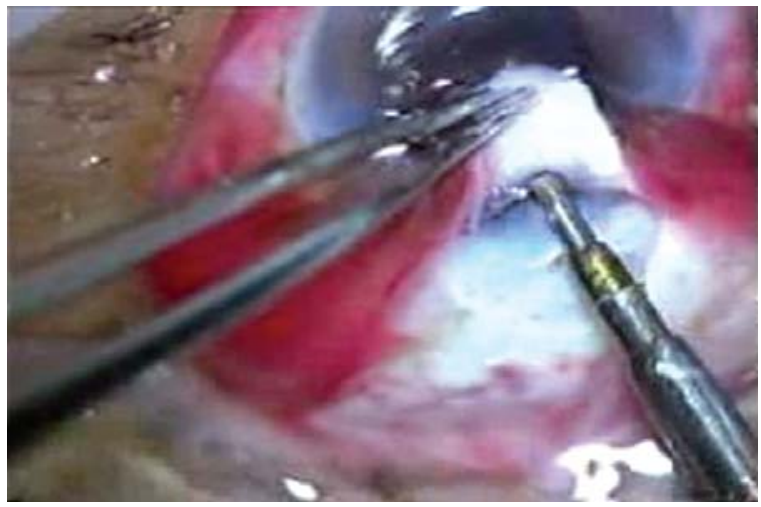

Fig. 4C: Trabeculectomy ostium being created using Kelly's descemet's punch

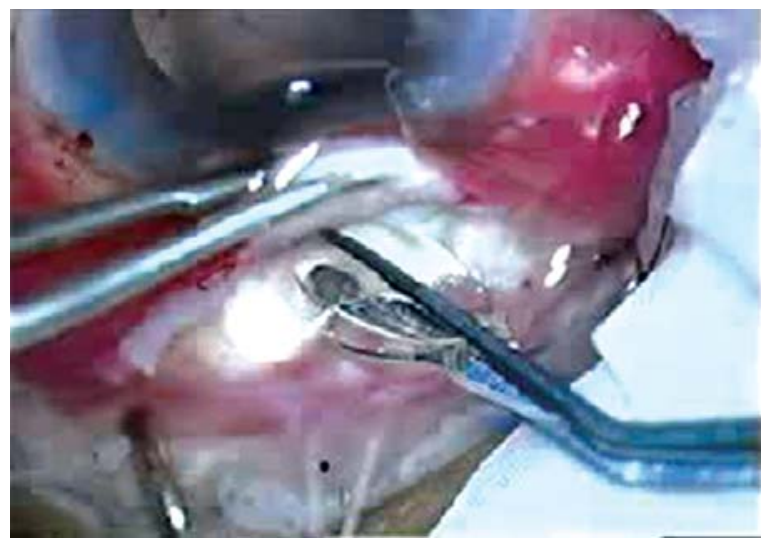

Fig. 4B: Insertion of foldable hydrophobic acrylic IOL through scleral flap

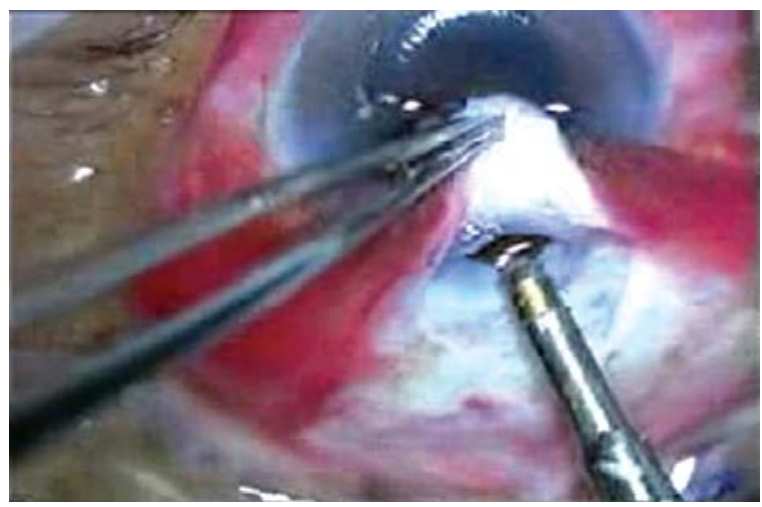

Fig. 4D: Trabeculectomy ostium

Figs 4A to D: MICS combined with trabeculectomy

has been suggested that the incidence of fibrinous reactions is greater following a combined procedure. This complication, usually encountered in the first three days following surgery is thought to be more frequent in eyes with myopia, ocular hypotension, hyphema, iridectomies, and exfoliation syndrome. The reported incidence ranges from $5-27 \%{ }^{29}$ The incidence appears to be less with phacoemulsification and foldable lens implantation.

2. Hyphema: The origin of this complication appears to be trauma to the iris or bleeding from the scleral flap. Usually it resolves within a week. More severe cases may mandate a surgical approach with bimanual irrigation- aspiration for hyphema drainage.

3. Vitreous loss: The incidence is similar to that following routine cataract surgery. However in this case, vitreous in the anterior chamber can block the internal ostium resulting in failure of the surgery. Hence recognizing this complication and a thorough anterior vitrectomy is advisable.
4. Elevated intraocular pressure: This is a common postoperative complication. Usually the internal ostium is blocked by retained viscoelastic, blood or fibrin. Blockade of the internal ostium may be diagnosed by gonioscopy. Other rare causes include a suprachoroidal hemorrhage and malignant glaucoma. A conservative management is advised. An attempt may be made to encourage filtration by a gentle digital massage on the inferior sclera through the lower lid. This method should not be used, when a fornix based conjunctival flap has been used to avoid leakage. Bleb formation is noted as digital massage forces aqueous out. If a dense fibrinous exudate is present, then intracameral tissue plasminogen activator may be used. Argon laser may be used to relieve the block in the ostium though occasionally surgical revision is required. In addition, a tight closure of the scleral flap may result in inadequate filtration. If releasable sutures have been used, they should be removed. The time for removal is within 2 weeks in routine 
surgery and within 6 weeks if Mitomycin C has been used intraoperatively. If two releaseable sutures have been used then one suture should be removed at a time. Additional digital massage may be needed to encourage filtration. Removal is not advised in the first week as this is associated with hypotony and leak from the anterior chamber. Removal is safe in the second and third weeks provided the conjunctiva is not too avascular and thinned out. Removal of sutures should also be considered if the conjunctiva overlying is excessively vascular.

5. Hypotony: Causes include a leaking bleb, overfiltration due to a large internal ostium, cyclodialysis cleft, and aqueous undersecretion due to iridocyclitis. The presence of a leaking bleb may be diagnosed by painting the suspected area with a fluorescein strip and examining the patient under the cobalt blue filter of the slit lamp. Such blebs are associated with an increased risk of infection. Management includes a pressure bandage, a large soft contact lens or scleral shell or cyanoacrylate glue. If all measures fail, resuturing of the conjunctiva may be required.

The use of antimetabolites is associated with hypotony. Usually the IOP tends to recover with time. Prolonged and untreated hypotony may lead to hypotony maculopathy and optic disk edema with a permanent drop in vision. This may require the use of a conjunctival autograft or a scleral graft to reinforce the area of the leak.

6. Failing blebs: Failed blebs are flat and vascular. Such blebs are best managed by needling. The procedure may be performed under the slit lamp or the operating microscope. $0.2 \mathrm{ml}$ of balanced salt solution is injected under the conjunctiva to elevate the conjunctiva to facilitate dissection. A 26G needle is inserted $1 \mathrm{~cm}$ from the bleb and sideways movement is used to dissect the scar tissue. ${ }^{30}$ The sclerostomy wound may be entered if need be. Success is indicated by a reduction in IOP and egress of aqueous with formation of the bleb. Antimetabolites are usually used at the end of the procedure. We prefer the use of 5-fluouracil $0.1 \mathrm{ml}$ of $50 \mathrm{mg} / \mathrm{ml}$ solution injected $1 \mathrm{~cm}$ from the bleb sites. Usually three injections are given on alternate days. 5-FU may be used alone without needling. Another option is to use topical mitomycin C soaked swab for 3 minutes over the bleb.

7. Use of iris hooks during surgery can lead to a permanent mydriasis and resultant postoperative glare and diplopia. Dilute pilocarpine therapy may be used initially although some patients may finally require a pupilloplasty with use of prolene sutures.

\section{CLINICAL STUDIES}

\section{Cataract Surgery in Patients with Glaucoma}

Many reports suggest that cataract surgery alone can decrease IOP in glaucoma patients. In an evidence-based review of the literature from 1964 to 2000, Jampel et al 11 stratified the IOP results from the studies abstracted into short-term (less than 24 hours) and long-term (more than 24 hours) results. The evidence for PECE ( phacoemulsification cataract extraction ) affecting short-term IOP was inconclusive, whereas the evidence that ECCE (extracapsular cataract extraction )elevated short-term IOP was weak. In contrast, although the evidence was graded as weak, the authors found an overall trend toward an IOP decrease of 2 to $4 \mathrm{~mm} \mathrm{Hg}$ in the long-term at 1 to 2 years after cataract extraction (both PECE and ECCE).

An evidence-based review on the effect of cataract surgery in eyes with prior filtering surgery concluded that the data up to the year 2000 were inconclusive as to whether or not cataract surgery had an adverse effect on filtering bleb function. ${ }^{31}$ One matched case control study in patients with prior filtering blebs undergoing clear cornea temporal PECE showed no IOP effect from cataract extraction based on survival analysis. In contrast, another study in patients with prior trabeculectomy who underwent ECCE found that approximately one-third of the patients required additional medical treatment or surgical management (including bleb needling or additional glaucoma surgery) to control IOP. ${ }^{32}$ One possible explanation for the difference in results is that the latter study included patients who underwent ECCE. Patients undergoing ECCE required additional glaucoma surgery for IOP control more frequently than patients who underwent PECE. These authors also identified iris manipulation during cataract extraction, age less than 50 years, preoperative IOP greater than $10 \mathrm{~mm} \mathrm{Hg}$, and postoperative IOP greater than $25 \mathrm{~mm} \mathrm{Hg}$ as risk factors for worse postoperative IOP control and bleb failure.

In a more recent prospective clinical trial, patients treated with trabeculectomy who demonstrated good IOP control without medications underwent PECE surgery and were followed for 1 year. After clear corneal PECE with posterior chamber IOL insertion, IOPs were significantly elevated 1 year postoperatively compared with baseline, and $34.7 \%$ of patients required glaucoma medications to control IOP.

However, the mean IOPs after phacoemulsification remained lower than the IOPs prior to trabeculectomy, and the number of required glaucoma medications required to control IOP were also lower than prior to trabeculectomy. Thus, the filtering surgery continued to have effect, but not as efficiently as before cataract extraction. This finding correlated with the observation that the filtering blebs decreased in size after cataract surgery. This decrease in function of the filtering blebs needs to be weighed against the significant improvement (up to 93.9\%) in visual acuity after cataract surgery.

The reasons for decreased bleb function after cataract surgery are not known, but most hypotheses center on postoperative inflammation and subconjunctival scarring in the area of the bleb. For these reasons, some surgeons have advocated using 5-fluorouracil (5-FU) injections and/or bleb 
needle revision routinely either at the time of cataract surgery and/or after cataract extraction to help maintain good filtering bleb function.

\section{Combined Glaucoma and Cataract Surgery}

Several recent evidence-based reviews of the glaucoma and cataract literature have addressed the outcomes of staged ${ }^{33,34}$ compared with combined cataract and glaucoma surgery. ${ }^{35-38}$ Summary analysis of key articles published up to the year 2000 related to the management of coexisting glaucoma and cataract revealed that insufficient evidence existed as to whether staged or combined cataract and glaucoma surgery was superior. In contrast, sufficient although weak evidence exists regarding the long-term IOP lowering effect of combined glaucoma and cataract surgery. Phacoemulsification cataract surgery with trabeculectomy reduced IOP by an average of $8 \mathrm{~mm} \mathrm{Hg}$ after 1 to 2 years of follow-up, whereas ECCE combined with trabeculectomy was slightly less effective, with an IOP lowering effect averaging 6 to $8 \mathrm{~mm} \mathrm{Hg}$ for a similar follow-up period.

However, trabeculectomy alone lowers the IOP more than combined filtering and PECE surgery.

More recent studies examining the role of mitomycin $\mathrm{C}$ or 5FU trabeculectomy with and without PECE also found that trabeculectomy alone produced greater IOP reduction than with combined surgery (58.5\% IOP reduction versus 31.5\%, respectively). ${ }^{39-42}$ Although the authors did not clarify the mechanisms that would explain these IOP differences, the explanation could be that the surgical trauma is higher in the combined surgery, and this causes a greater alteration of the blood-aqueous barrier or an increase of transforming growth factor beta. The literature-based evidence is even stronger that ECCE combined with trabeculectomy produces less IOP control long-term than does filtering surgery alone. Thus, the decision to perform cataract surgery in combination with glaucoma surgery needs to take into account the risk of diminished longterm IOP lowering with glaucoma surgery in contrast to the potential improved quality of life with cataract extraction.

The trabeculectomy conjunctival flap can be either fornix or limbal based. Both flap types result in similar levels of IOP control, ${ }^{43-46}$ although fornix-based conjunctival flaps have a higher incidence of limbal bleb leaks if the limbal conjunctiva is not carefully closed.

Another important aspect of combined surgery is the decision between one or two sites. ${ }^{47,48}$ There is no strong evidence to demonstrate that one technique is better than the other, although the data lean toward the two-site technique.

Friedman et al reported slightly better results when the incisions were created separately. They quantified the effect as an additional improvement of 1 to $2 \mathrm{~mm} \mathrm{Hg}$, similar to reports by el Sayyad et al. ${ }^{35}$ Wyse et $\mathrm{al}^{36}$ concluded that when both techniques were compared in 33 eyes, the IOP values were similar but the eyes treated with one-site surgery required more medication than the eyes treated with two-site surgery. Jampel et $\mathrm{al}^{11}$ also noted the superiority of the two-site technique over the one-site, reporting an IOP difference of 1 to $2 \mathrm{~mm} \mathrm{Hg}$. Jampel et $\mathrm{al}^{11}$ further noted that 5 -fluorouracil does not further reduce IOP when combined surgery is performed, while IOP reduction between 2 and $4 \mathrm{~mm} \mathrm{Hg}$ is noted with the use of MMC.

In a recent retrospective comparative study Shingleton et $\mathrm{al}^{48}$ reviewed case records of patients that underwent single site (71 eyes) or two site (64 eyes) phacotrabeculectomy and concluded that there was no statistically significant difference in the achievement of best corrected visual acuity (BCVA), IOP control over one year and the requirement of antiglaucoma medications in the two groups over the study period of one year.

The use of mitomycin $\mathrm{C}$ during phacotrabeculectomy was also evaluated by Park et $\mathrm{al}^{49}$ in patients with uveitis in a retrospective study over a period of 1-2 years. They demonstrated that the success rate for long-term IOP control was significantly lesser in uveitic eyes than in nonuveitic eyes even when the postoperative visual acuities were not significantly different in the 2 groups. The failure rate was correlated with the occurrence of postoperative recurrence of inflammation in the uveitis eyes.

In a recent retrospective comparison between phacotrabeculectomy with MMC vs trabeculectomy MMC, Murthy et $\mathrm{al}^{50}$ showed that over two years similar proportion of patients from both the groups achieved their target IOPs. However trabeculectomy with MMC was associated with more IOP reduction from the baseline than phacotrabeculectomy over the entire study period.

The refractive outcomes following phacotrabeculectomy were compared with that following trabeculectomy in a recent series by Chan JC et al. ${ }^{51}$ They found that phacotrabeculectomy was more likely to have a prediction error $>1.00 \mathrm{D}(\mathrm{P}=0.02)$ and a myopic shift of $>0.50 \mathrm{D}$ or $1.00 \mathrm{D}$ ( $\mathrm{P}=0.03$ or 0.02 , respectively). No significant differences were found with regard to the frequency of hyperopic shift of $>0.5 \mathrm{D}$.

In a head to head comparison of the use of different types of IOLs during phacotrabeculectomy, Serpa Junior E et al ${ }^{52}$ compared PMMA, foldable silicon and hydrophobic acrylic IOLs during phacotrabeculectomy. Even though there was no statistically significant difference between the BCVAs in the three groups over 12 month study period, the silicone group had significantly more postoperative fibrin reaction into the anterior chamber $(p=0.01)$ and giant cell deposits on the IOL ( $<<0.0001)$ than the PMMA and the acrylic groups. The rate of YAG laser posterior capsulotomy was lower with the acrylic IOL (0\%) than with the silicone (12.2\%) or PMMA (13.3\%) IOLs $(\mathrm{p}=0.08)$. 


\section{CONCLUSION}

Combined cataract and filtering surgery is a feasible and successful approach to treat coexisting glaucoma and cataract. The procedure has got a learning curve but once the surgeon has mastered both the techniques of phacoemulsification and filtering surgery, then he can safely perform the combined procedure with confidence. The success rates being lower than filtering surgery alone, treatment needs to be individualized for each patient. There are increased rates of postoperative reaction and posterior capsular opacification following the combined procedure. Patients adequately controlled by medication/ previous successful surgery may be rehabilitated by cataract surgery alone. Phacoemulsification is the preferred technique to remove cataract and MICS (Phakonit) may be preferred while performing combined surgery, although the technique is still evolving. Patients undergoing a combined surgery benefit from use of Mitomycin C with a greater IOP reduction. All patients should be maintained on a long-term follow-up with regular assessment of the intraocular pressure, optic disk and visual fields as the long-term success and the IOP control achieved after a phacotrabeculectomy is less than that achieved with a standard trabeculectomy. The technique of phacotrabeculectomy once mastered can provide gratifying results both in terms of improvement of visual acuity as well as maintenance of IOP in the long-term without the morbidity of two separate surgical procedures.

\section{REFERENCES}

1. Friedman DS, Jampel HD, Lubomski LH, Kempen JH, Quigley H, Congdon N, Verbin HL, Robinson KA, Bass EB. Surgical Strategies for Coexisting Glaucoma and Cataract. Ophthalmology 2002;109:1902-13.

2. Lee RK, Gedde SJ. Surgical Management of Coexisting Cataract and Glaucoma. Int Ophthalmol Clin 2004;44:151-66.

3. Verges C, Jorge C, Cosme L. Surgical strategies in patients with cataract and glaucoma. Curr Opin Ophthalmol 2005;16:44-52.

4. Aggarwal S, Sundaram, Asha B. Laser Sclerotomy, Laser Phakonit and IOL Implantation. In: Agarwal S, Agarwal A (Eds). Phacoemulsification. New Delhi: Jaypee Brothers 2004;60912.

5. Agarwal A, Agarwal S, et al. Phakonit: Phacoemulsification through a $0.9 \mathrm{~mm}$ corneal incision. J Cataract Refract Surg 2001;27:1548-52.

6. Agarwal A, Agarwal S. Phakonit. In: Agarwal S, Agarwal A (Eds). Phacoemulsification. New Delhi: Jaypee Brothers 2004;1:318-29.

7. Tsuneoka H, Shiba T, Talcehashi Y. Ultrasonic phacoemulsification using a $1.4 \mathrm{~mm}$ incision. J Cataract Refract Surg 2002;28:8186.

8. Soscia W, Howard JG, Olson RJ. Microphacoemulsification with Whitestar: A wound temperature study. J Cataract Refrac Surg 2002;28:1044-46.
9. Hoffman KB, Feldman RM, Budenz DL, Gedde SJ, Chacra GA, Schiffman JC. Combined Cataract Extraction and Baerveldt Glaucoma Drainage Implant. Ophthalmology 2002;109:191620.

10. Casson RJ, Salmon JF. Combined surgery in the treatment of patients with cataract and primary open-angle glaucoma. J Cataract Refract Surg 2001;27:1854-63.

11. Jampel HD, Friedman DS, Lubomski LH, Kempen JH, Quigley H, Congdon N, Verbin HL, Robinson KA, Bass EB. Effect of technique on intraocular pressure after combined cataract and glaucoma surgery. Ophthalmology 2002;109:2215-24.

12. Donoso R, Rodrfguez. Combined versus sequential phacotrabeculectomy with intraoperative 5-fluoracil. J Cataract Refract Surg 2000;26:71-74.

13. Cohen JS, Khatana AK, Osher RH. Combined Cataract Implant and Filtering Surgery. In:Steinert RF (Ed). Cataract Surgery. Philadelphia: Saunders 2004;223-46.

14. Beckers HJ, De Kroon KE, Nuijts RM, Webers CA. Phacotrabeculectomy. Doc Ophthalmol 2000;100:43-47.

15. Vass C, Menapace R. Surgical strategies in patients with combined cataract and glaucoma. Curr Opin Ophthalmol 2004;15:61-66.

16. Caprioli J, Park HJ, Weitzman M. Temporal corneal phacoemulsification combined with superior trabeculectomy: A controlled study. Trans Am Ophthalmol Soc 1996;94:451-63; discussion 463-68.

17. Dada T, Muralidhar R, Sethi HS. MICS combined with trabeculectomy. In Garg A, Fine H, Chang DF (Eds). Mastering the art of Bimanual Microincision Phacoemulsification. Jaypee Brothers Medical Publishers. New Delhi 2005;346-54.

18. Gayton JL, Karr MVD, Sanders V. Combined cataract and glaucoma surgery: Trabeculectomy versus endoscopic laser cycloablation. J Cataract Refract Surg 1999;25:1214-19.

19. Rebolleda G, Munoz-Negrete FJ. Phacoemulsification in eyes with functioning filtering blebs: A prospective study. Ophthalmology 2002;109:2248-55.

20. Chen PP, Weaver YK, Budenz DL, et al. Trabeculectomy function after cataract extraction. Ophthalmology 1998;105:1928-35.

21. Dogru M, Omoto M, Fujishima H, Yagi Y, Tsubota K. Early visual results with rollable ThinOptx intraocular lens. J Cat Refract Surg 2004;30:558-65.

22. Dada T, Muralidhar R, Sethi HS. Insertion of a foldable hydrophobic IOL through the trabeculectomy fistula in cases with Microincision cataract surgery combined with trabeculectomy. BMC Ophthalmol 2006;19(6):14.

23. Diaz-Valle D, del Castillo B, Jimenez A, et al. Endothelial changes with cataract surgery techniques. J Cataract Refract Surg 1998;24:951-55.

24. Milla E, Verges C, Cipres MC. Corneal endothelium evaluation after phacoemulsification with continuous anterior chamber infusion. Cornea 2005;24:278-82.

25. Tsuneoka H, Shiba T, Talcehashi Y. Ultrasonic phacoemulsification using a $1.4 \mathrm{~mm}$ incision. J Cataract Refract Surg 2002;28:8186.

26. Verges C, Cazal J, Cipres MC, et al. Endothelial cell density and corneal thickness in cataract surgery comparing continuous vs micropulsed ultrasound. Cornea (Epub ahead of print). 
27. Fine H, Packer M, Hoffman RS: Power modulation in new phacoemulsification technology: Improved outcomes. J Cataract Refract Surg 2004;30:1014-19.

28. Gosiengfiao DH, Latina MA. Avoiding complications in combined phacotrabeculectomy. Semin Ophthalmol Sep-Dec 2002;17(3-4):138-43.

29. Siriwardena D, Kotecha A, Minassian D, et al. Anterior chamber flare after trabeculectomy and after phacoemulsification. Br J Ophthalmol 2000;84:1056-57.

30. Greenfield DS, Miller MP, Suner IJ, et al. Needle elevation of the scleral flap for failing filtration blebs after trabeculectomy with mitomycin C. Am J Ophthalmol 1996;122:195-204.

31. Mietz H, Andresen A, Welsandt G, Krieglstein GK. Effect of cataract surgery on intraocular pressure in eyes with previous trabeculectomy. Graefes Arch Clin Exp Ophthalmol 2001;239:763-69.

32. Drolsum L, Haaskjold E. Extracapsular cataract extraction in eyes previously operated for glaucoma. Acta Ophthalmol (Copenh) 1994;72:273-78.

33. Lochhead J, Casson RJ, Salmon JF. Long-term effect on intraocular pressure of phacotrabeculectomy compared to trabeculectomy. Br J Ophthalmol 2003;87:850-52.

34. Kleinmann G, Katz H, Pollack A, et al. Comparison of trabeculectomy with mitomycin $\mathrm{C}$ with or without phacoemulsification and lens implantation. Ophthalmic Surg Lasers 2002;33:102-08.

35. El Sayyad F, Helal M, el Maghraby A, et al. One-site versus two-site phacotrabeculectomy: A randomized study. J Cataract Refract Surg 1999;25:77-82.

36. Wyse T, Meyer M, Ruderman JM, et al. Combined trabeculectomy and phacoemulsification: A one-site vs two-site approach. Am J Ophthalmol 1998;125:334-39.

37. Caporossi A, Casprini F, Tosi GM, Balestrazzi A. Long-term results of combined 1-way phacoemulsification, intraocular lens implantation, and trabeculectomy. J Cataract Refract Surg 1999;25:1641-45.

38. Rossetti L, Bucci L, Miglior S, et al. Temporal corneal phacoemulsification combined with separate-incision superior trabeculectomy vs standard phacotrabeculectomy: A comparative study. Acta Ophthalmol Scand (Suppl) 1997;224:39.

39. Cohen JS: Combined cataract implant and filtering surgery with 5-fluorouracil. Ophthalmic Surg 1990;21:181-86.

40. Budenz DL, Pyfer M, Singh K, et al. Comparison of phacotrabeculectomy with 5-fluorouracil, mitomycin $\mathrm{C}$ and without antifibrotic agents. Ophthalmic Surg Lasers 1999;30:36774.

41. Chang L, Thiagarajan M, Moseley M, Woodruff S, Bentley C, Khaw PT, Bloom P. Intraocular pressure outcome in primary 5FU phacotrabeculectomies compared with 5FU trabeculectomies. J Glaucoma Dec 2006;15(6):475-81.

42. Shin DH, Ren J, Juzych MS, et al. Primary glaucoma triple procedure in patients with primary open-angle glaucoma: The effect of mitomycin $\mathrm{C}$ in patients with and without prognostic factors for filtration failure. Am J Ophthalmol 1998;125:34652.

43. Shingleton BJ, Chaudhry IM, O'Donoghue MW, et al. Phacotrabeculectomy: Limbus-based versus fornix-based conjunctival flaps in fellow eyes. Ophthalmology 1999;106:1152-55.

44. Lemon LC, Shin DH, Kim C, et al. Limbus-based vs fornixbased conjunctival flap in combined glaucoma and cataract surgery with adjunctive mitomycin C. Am J Ophthalmol 1998;125:34045 .

45. Kozobolis VP, Siganos CS, Christodoulakis EV, et al. Two-site phacotrabeculectomy with intraoperative mitomycin-C: Fornixversus limbus-based conjunctival opening in fellow eyes. $\mathrm{J}$ Cataract Refract Surg 2002;28:1758-62.

46. Berestka JS, Brown SV. Limbus- versus fornix-based conjunctival flaps in combined phacoemulsification and mitomycin C trabeculectomy surgery. Ophthalmology 1997;104:187-96.

47. Borggrefe L, Lieb W, Grehn F. A prospective randomized comparison of two techniques of combined cataract-glaucoma surgery. Graefes Arch Clin Exp Ophthalmol 1999;237:887-92.

48. Shingleton BJ, Price RS, O'Donoghue MW, Goyal S. Comparison of 1-site versus 2-site phacotrabeculectomy. J Cataract Refract Surg 2006;32:799-802.

49. Park UC, Ahn JK, Park KH, Yu HG. Phacotrabeculectomy with mitomycin $\mathrm{C}$ in patients with uveitis. Am J Ophthalmol 2006;142:1005-12.

50. Murthy SK, Damji KF, Pan Y, Hodge WG. Trabeculectomy and phacotrabeculectomy, with mitomycin-C, show similar twoyear target IOP outcomes. Can J Ophthalmol 2006;41:51-59.

51. Chan JC, Lai JS, Tham CC. Comparison of postoperative refractive outcome in phacotrabeculectomy and phacoemulsification with posterior chamber intraocular lens implantation. J Glaucoma 2006;15:26-29.

52. Serpa Junior E, Wishart PK. Comparison of PMMA, foldable silicone and foldable acrylic hydrophobic intraocular lenses in combined phacoemulsification and trabeculectomy. Arq Bras Oftalmol 2005;68:29-35.

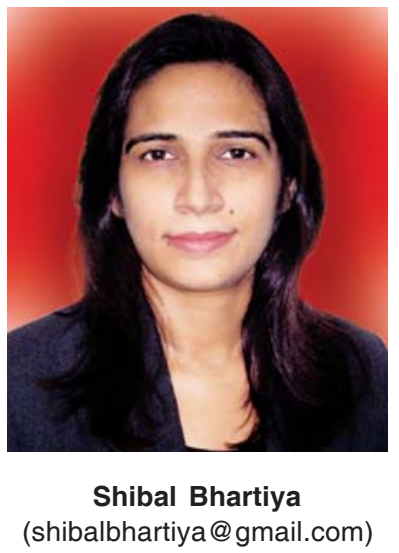

Learning is the beginning of wealth. Learning is the beginning of health. Learning is the beginning of spiritually. Searching and learning is where the miracle process all begins. 